\title{
Increased plasma levels of damage-associated molecular patterns during systemic anticancer therapy in patients with advanced lung cancer
}

\author{
Hiroyuki Inoue ${ }^{1,2,3 \#}$, Hirono Tsutsumi ${ }^{1 \#}$, Kentaro Tanaka ${ }^{1}$, Eiji Iwama ${ }^{1}$, Yoshimasa Shiraishi ${ }^{1}$, \\ Aiko Hirayama ${ }^{1}$, Takayuki Nakanishi ${ }^{1}$, Hiroyuki Ando ${ }^{1}$, Maako Nakajima ${ }^{1}$, Seiji Shinozaki ${ }^{1}$, \\ Hiroaki Ogata ${ }^{1}$, Kazuyasu Uryu ${ }^{1}$, Koji Okamura ${ }^{1}$, Shinichi Kimura ${ }^{1}$, Tomohiro Ogawa ${ }^{1}$, Keiichi Ota ${ }^{1}$, \\ Yasuto Yoneshima ${ }^{1}$, Naoki Hamada ${ }^{1}$, Yoichi Nakanishi ${ }^{1,2}$, Isamu Okamoto ${ }^{1}$ \\ ${ }^{1}$ Research Institute for Diseases of the Chest, Graduate School of Medical Sciences, Kyushu University, Fukuoka, Japan; ${ }^{2}$ Center for Clinical and \\ Translational Research, Kyushu University Hospital, Fukuoka, Japan; ${ }^{3}$ Department of Respiratory Medicine, Fukuoka University School of Medicine, \\ Fukuoka, Japan \\ Contributions: (I) Conception and design: H Inoue; (II) Administrative support: H Inoue, I Okamoto, Y Nakanishi; (III) Provision of study materials \\ or patients: H Tsutsumi, Y Shiraishi, A Hirayama, T Nakanishi, H Ando, M Nakajima, S Shinozaki, H Ogata, K Uryu, K Okamura, S Kimura, T \\ Ogawa, K Tanaka, E Iwama, K Ota, Y Yoneshima, N Hamada; (IV) Collection and assembly of data: H Tsutsumi, K Tanaka, Y Yoneshima; (V) Data \\ analysis and interpretation: H Inoue, H Tsutsumi; (VI) Manuscript writing: All authors; (VII) Final approval of manuscript: All authors. \\ \#These authors contributed equally to this work. \\ Correspondence to: Dr. Isamu Okamoto. Research Institute for Diseases of the Chest, Graduate School of Medical Sciences, Kyushu University, 3-1-1 \\ Maidashi, Higashi-ku, Fukuoka 812-8582, Japan. Email: okamoto.isamu.290@m.kyushu-u.ac.jp.
}

Background: Immunogenic cell death (ICD) characterized by the release of damage-associated molecular patterns (DAMPs) from dying cancer cells may contribute to the synergistic antitumor effect of cytotoxic chemotherapy combined with an immune checkpoint inhibitor. The kinetics of circulating DAMP levels in cancer patients have remained largely uncharacterized, however.

Methods: We evaluated the possible effects of various systemic anticancer therapy modalities on the kinetics of plasma DAMP concentrations in a prospective observational study of patients with advanced lung cancer. The plasma concentrations of high-mobility group box 1 (HMGB1), calreticulin (CRT), heat shock protein 70 (HSP70), annexin A1, and histone H3 were thus determined in 121 such patients at four time points during the first cycle of treatment.

Results: The mean of the maximum fold change in HMGB1, HSP70, or annexin A1 concentration observed during treatment was significantly greater than the corresponding baseline value $(\mathrm{P}<0.005)$. The maximum fold changes in HMGB1 and CRT concentrations tended to be associated with clinical response as evaluated by RECIST criteria, although the changes in the levels of these two DAMPs were not correlated, suggestive of differential induction mechanisms. Among the various treatment modalities administered, platinum-based combination or single-agent chemotherapy tended to elicit robust increases in the concentrations of HMGB1 and CRT.

Conclusions: Serial monitoring of plasma revealed that systemic anticancer therapy increased the circulating levels of HMGB1 and CRT and that these changes tended to be associated with clinical response, suggesting that agents capable of releasing these DAMPs into plasma might induce ICD in advanced lung cancer patients.

Keywords: Immunogenic cell death (ICD); damage-associated molecular pattern (DAMP); high-mobility group box 1 (HMGB1); calreticulin; lung cancer

Submitted Feb 04, 2021. Accepted for publication Mar 30, 2021.

doi: $10.21037 /$ tlcr-21-92

View this article at: http://dx.doi.org/10.21037/tlcr-21-92 


\section{Introduction}

Lung cancer is the leading cause of cancer-related mortality worldwide and has a poor prognosis (1). The standard treatments for advanced non-small cell lung cancer and small cell lung cancer have changed markedly over the last decade, however (2). Several recent phase 3 studies have revealed that platinum (cisplatin or carboplatin)based combination chemotherapy together with immune checkpoint inhibitors including antibodies to programmed cell death-1 (PD-1) or to its ligand PD-L1 confers a significantly longer overall survival compared with chemotherapy alone in individuals with advanced lung cancer (3-6). Such synergistic effects have been proposed to be attributable in part to the induction of immunogenic cell death (ICD) (7), a type of regulated cell death that results in the activation of a secondary adaptive immune response to tumor-associated antigens. ICD has been characterized by the release or exposure on the cell surface of a defined set of molecules that are known as damage-associated molecular patterns (DAMPs) $(8,9)$ and which include highmobility group box 1 (HMGB1), calreticulin (CRT), heat shock protein 70 (HSP70), and annexin A1 $(8,10)$. Histone $\mathrm{H} 3$ has also been shown to function as a DAMP, or alarmin, in inflammatory conditions $(11,12)$. Recent preclinical studies have indicated that several chemotherapeutic agents stimulate an antitumor immune response by triggering DAMP-related ICD in addition to exerting direct cytotoxicity $(7,13-15)$.

An approach to evaluation of ICD in cancer patients undergoing anticancer therapy has not been well established (9). Although immunohistochemical detection of DAMPs as ICD-associated biomarkers in surgically resected tumor specimens has provided prognostic indications under certain circumstances $(16,17)$, this procedure is not applicable to patients with inoperable or recurrent tumors and is not suitable for repeat assessments. The establishment of an approach to the detection of surrogate ICD biomarkers that is safer, easier to perform, and more amenable to repeated evaluation is thus urgently needed. As such an approach, we have focused on sampling of peripheral blood for measurement of DAMPs in patients with advanced lung cancer. Evidence for the practicality of this approach has been provided by the previous findings that an enzyme-linked immunosorbent assay (ELISA) was able to detect a preoperative chemoradiation-induced increase in the serum level of HMGB1 in patients with esophageal squamous carcinoma (17), and that the serum concentration of CRT was higher in patients with lung cancer (18) or rheumatoid arthritis (19) than in healthy control individuals. However, little information has been available with regard to the kinetics of DAMP levels, especially those in peripheral blood during the early phase of anticancer therapy in advanced cancer patients, as well as with regard to the relation of such kinetics to clinical response.

We have now conducted a prospective observational study to investigate the possible effects of systemic anticancer therapy-including platinum-based combination chemotherapy, concurrent chemoradiotherapy (CCRT), single-agent chemotherapy, and molecularly targeted therapy [tyrosine kinase inhibitors (TKIs) specific for the epidermal growth factor receptor (EGFR) or anaplastic lymphoma kinase (ALK)] — on the plasma levels of DAMPs including HMGB1, CRT, HSP70, annexin A1, and histone $\mathrm{H} 3$ in 121 individuals with advanced lung cancer. We also examined the relation between the extent of any such changes and clinical outcome.

We present the following article in accordance with the STROBE reporting checklist (available at http://dx.doi. org/10.21037/tlcr-21-92).

\section{Methods}

\section{Patients and sample collection}

We conducted a prospective observational study of treatment-naïve or previously treated patients with advanced (stage III or IV according to the 8th edition of the TNM classification) or recurrent lung cancer who underwent a first cycle of systemic anticancer therapy-including cytotoxic chemotherapy [such as platinum (cisplatin or carboplatin) doublet chemotherapy and single-agent chemotherapy (including docetaxel, pemetrexed, and S-1 among others)], CCRT (cisplatin- or carboplatin-based), or molecularly targeted therapy with an EGFR-TKI or ALKTKI-at Kyushu University Hospital between February 2019 and March 2020. The concentrations of DAMPs were measured in plasma collected at four time points: immediately before and on days 3 and 8 after the onset of the first cycle of treatment as well as immediately before the second treatment cycle. The best clinical response observed after the first or second cycle of treatment was defined according to the Response Evaluation Criteria in Solid Tumors (RECIST, version 1.1). The study was conducted in accordance with the Declaration of Helsinki (as revised 
Table 1 Characteristics and treatment regimens of the study patients $(\mathrm{n}=121,100 \%)$

\begin{tabular}{|c|c|}
\hline Characteristic & n [\%] \\
\hline Median age [range], years & $67[31-89]$ \\
\hline \multicolumn{2}{|l|}{ Sex } \\
\hline Male & 88 [73] \\
\hline Female & $33[27]$ \\
\hline \multicolumn{2}{|l|}{ Smoking history } \\
\hline Former or current & $100[83]$ \\
\hline Never & $21[17]$ \\
\hline \multicolumn{2}{|l|}{ ECOG performance status } \\
\hline 0 & $26[21]$ \\
\hline 1 & 88 [73] \\
\hline 2 or 3 & $7[6]$ \\
\hline \multicolumn{2}{|l|}{ Histology } \\
\hline Adenocarcinoma & $70[58]$ \\
\hline Squamous cell carcinoma & $18[15]$ \\
\hline NOS & 9 [7] \\
\hline Small cell lung cancer & $24[20]$ \\
\hline \multicolumn{2}{|l|}{ Oncogenic driver } \\
\hline EGFR mutation & $11[9]$ \\
\hline$A L K$ rearrangement & $4[3]$ \\
\hline \multicolumn{2}{|l|}{ Clinical stage at screening } \\
\hline III & 38 [31] \\
\hline IV & 83 [69] \\
\hline \multicolumn{2}{|c|}{ Number of prior treatment lines at screening } \\
\hline 0 & $81[67]$ \\
\hline 1 & 15 [12] \\
\hline$\geq 2$ & $25[21]$ \\
\hline \multicolumn{2}{|l|}{ Systemic anticancer therapy regimens } \\
\hline Platinum doublet chemotherapy & 59 [49] \\
\hline Single-agent chemotherapy & 28 [23] \\
\hline CCRT & 23 [19] \\
\hline EGFR-TKI or ALK-TKI & $11[9]$ \\
\hline
\end{tabular}

ECOG, Eastern Cooperative Oncology Group; NOS, not otherwise specified; EGFR, epidermal growth factor receptor; ALK, anaplastic Iymphoma kinase; CCRT, concurrent chemoradiotherapy; TKI, tyrosine kinase inhibitors. in 2013). It was approved by the Ethics Committee of Kyushu University and Kyushu University Hospital (IRB: 30-434), and informed consent was taken from all individual participants.

\section{ELISAs}

The concentration of HMGB1 in plasma was measured with an ELISA kit (\#326054329; Shino-Test Corp., Sagamihara-shi, Japan) (20). The plasma concentration of histone $\mathrm{H} 3$ was also measured with an ELISA kit (ShinoTest Corp.) as recently described $(21,22)$. The plasma levels of CRT (\#ELH-CALR-1; RayBiotech, Peachtree Corners, GA), HSP70 (\#KE00059; Proteintech, Tokyo, Japan), and annexin A1 (\#EK1745; Boster Biological Technology, Pleasanton, CA) were similarly quantified with ELISA kits.

\section{Statistical analysis}

Statistical analysis was performed with GraphPad Prism 5.0d software (GraphPad Software, San Diego, CA; RRID: SCR_002798). Changes in plasma DAMP levels were evaluated with the paired $t$ test, one-way analysis of variance (ANOVA), repeated-measures ANOVA, or Pearson's correlation analysis, as indicated. A $\mathrm{P}$ value of $<0.05$ was considered statistically significant.

\section{Results}

\section{Patient characteristics}

A total of 121 individuals with advanced lung cancer scheduled to receive systemic anticancer therapy was enrolled. The baseline characteristics of the study patients are shown in Table 1. The median age was 67 years (range, 31-89 years), and 88 (73\%) patients were male. Seventy (58\%) patients had adenocarcinoma, 18 (15\%) squamous cell carcinoma, and $24(20 \%)$ small cell lung cancer, and $83(69 \%)$ patients had stage IV disease. With regard to treatment modalities, 59 (49\%) patients received platinum (cisplatin or carboplatin) doublet chemotherapy, 28 (23\%) single-agent chemotherapy (docetaxel, pemetrexed, or S-1 among others), 23 (19\%) CCRT (cisplatin- or carboplatin-based), and 11 (9\%) an EGFR-TKI (erlotinib or osimertinib) or ALK-TKI (alectinib, crizotinib, or 
brigatinib).

\section{Increased plasma DAMP levels during systemic anticancer therapy}

We measured the plasma levels of five DAMPs (HMGB1, CRT, HSP70, annexin A1, and histone $\mathrm{H} 3$ ) at four serial time points including baseline (the day before the first cycle of systemic anticancer therapy), days 3 and 8 of the first cycle, and the day before the second cycle of treatment (Figure S1). The fold changes in the concentrations of the five DAMPs during the first treatment cycle relative to the baseline value were determined (Figure 1A), with the fold changes in both HMGB1 and CRT levels at the three time points after treatment onset being substantially higher than the baseline value but those in HSP70 and annexin A1 being only slightly higher (Figure $1 B$ ). The mean of the maximum fold change in HMGB1, HSP70, or annexin A1 apparent after the onset of systemic anticancer therapy was significantly higher than that of the corresponding baseline value $[1.00$ vs. $3.15(\mathrm{P}=0.002), 1.00$ vs. 1.57 $(\mathrm{P}<0.0001)$, and 1.00 vs. $1.18(\mathrm{P}<0.0001)$, respectively] (Figure 1C). No corresponding significant difference was apparent for CRT and histone H3 levels [1.00 vs. 6.52 $(\mathrm{P}=0.053)$ and 1.00 vs. $1.26(\mathrm{P}=0.60)$, respectively].

\section{Association between changes in plasma DAMP levels and clinical response}

We next evaluated the possible association between the maximum fold changes in plasma DAMP concentrations relative to baseline and clinical response as determined by RECIST criteria. Given that histone $\mathrm{H} 3$ was undetectable in plasma of 97 patients at baseline, it was excluded from this analysis. The maximum fold changes in both HMGB1 and CRT levels for patients showing a complete or partial response were higher than those for patients showing stable disease [means $\pm \mathrm{SEM}$ of $3.98 \pm 1.68$ vs. $2.83 \pm 0.61(\mathrm{P}=0.75$, Student's $t$ test) and $13.06 \pm 8.13$ vs. $3.56 \pm 1.46(\mathrm{P}=0.28)$, respectively], which were in turn higher than those for patients with progressive disease $[2.83 \pm 0.61$ vs. $1.68 \pm 0.18$ $(\mathrm{P}=0.86)$ and $3.56 \pm 1.46$ vs. $1.93 \pm 0.36(\mathrm{P}=0.98)$, respectively] (Figure $2 A, B$ ), whereas no such substantial differences were apparent for HSP70 and annexin A1 concentrations (Figure $2 C, D$ ). Although these results were not statistically significant, they suggested that clinical response might be associated with the maximum fold change in plasma levels of HMGB1 and CRT.

\section{Comparison of the maximum fold change in DAMP levels among systemic anticancer therapy modalities}

To identify the most effective inducers of ICD among systemic anticancer therapy modalities, we next compared the maximum fold changes in plasma DAMP levels for patients showing a complete $(n=1)$ or partial $(n=43)$ response. The mean values for the maximum fold change in HMGB1 levels were numerically greater in patients receiving platinum-based combination or single-agent chemotherapy than in those receiving CCRT or TKIs (Figure $3 A$ ). The maximum fold change in plasma CRT levels was greatest in patients treated with platinum-based combination chemotherapy (Figure 3B). There were no robust differences in the maximum changes in HSP70 or annexin A1 levels among treatment modalities (Figure 3C,D).

\section{Correlation between maximum fold changes in plasma DAMP levels}

Finally, we performed a correlation analysis for the maximum fold changes in the levels of HMGB1, CRT, HSP70, and annexin A1 in the total patient population $(\mathrm{n}=121)$. There were no significant correlations between maximum fold changes for HMGB1 versus those for CRT, HSP70, or annexin A1 (Figure $4 A, B, C$ ). In contrast, there was a significant correlation between maximum fold changes for HSP70 and either CRT or annexin A1, although not between those for CRT and annexin A1 (Figure 4D,E,F). Rates of concordance were examined for patients who showed a $>2$-fold increase in HMGB1 $(\mathrm{n}=34)$, CRT $(\mathrm{n}=34)$, or HSP70 $(\mathrm{n}=24)$ levels (Figure $4 G$ ), with this analysis not being performed for annexin A1 because only five patients showed such an increase. The rates of concordance for HMGB1, CRT, and HSP70; for HMGB1 and CRT; for HMGB 1 and HSP70; and for CRT and HSP70 were $11.3 \%, 21.0 \%, 24.2 \%$, and $14.5 \%$, respectively.

\section{Discussion}

We have here shown that the plasma concentrations of HMGB1, CRT, HSP70, and annexin A1 were substantially increased during anticancer therapy in a subset of individuals with advanced lung cancer. We also found that the extent of the change in the plasma levels of HMGB1 or CRT tended to be associated with clinical response, 
A

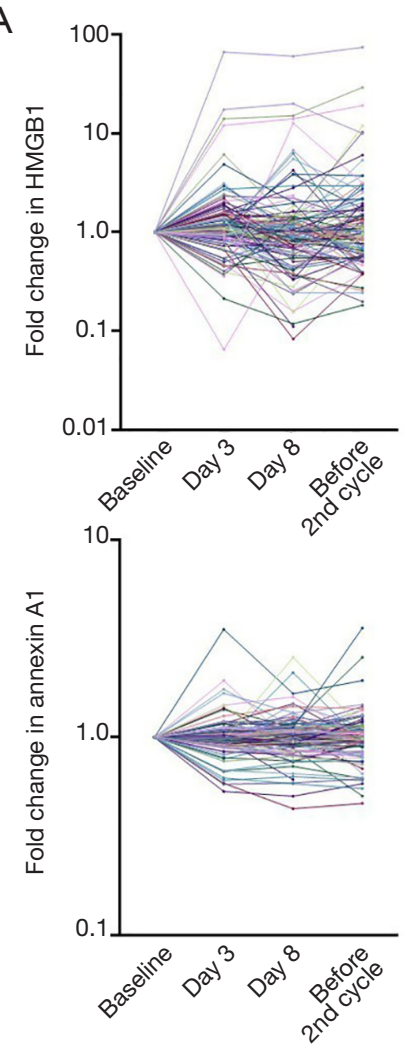

B
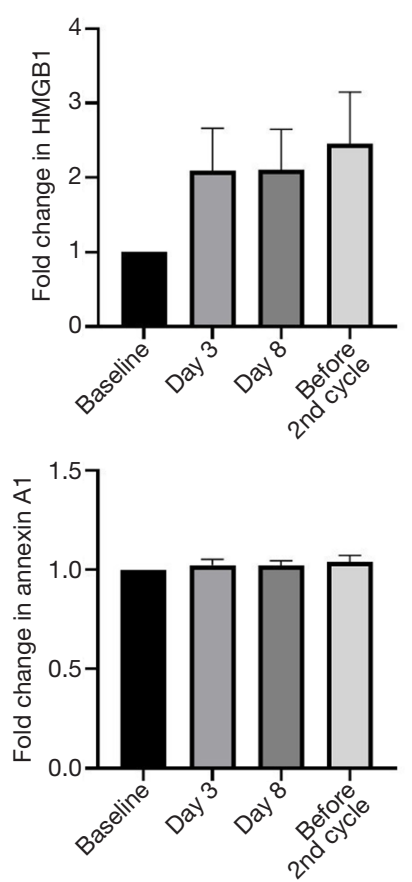
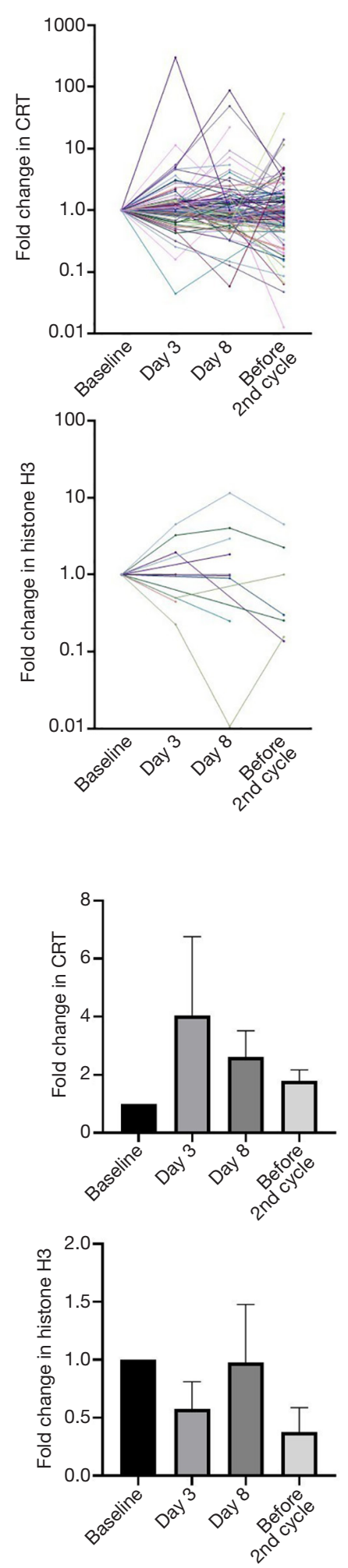
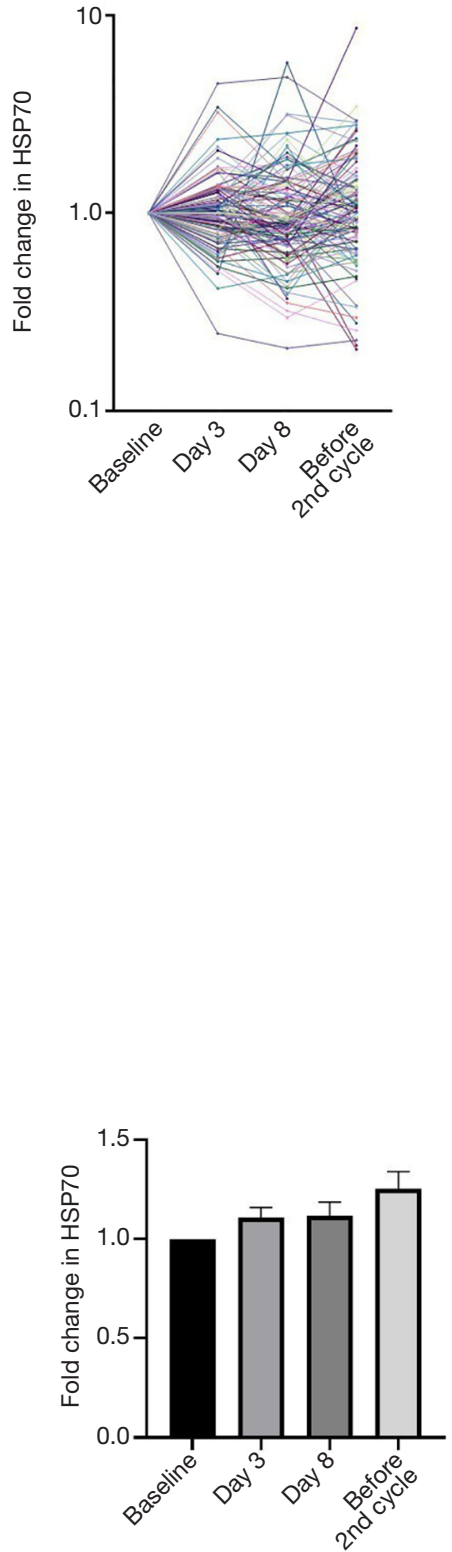

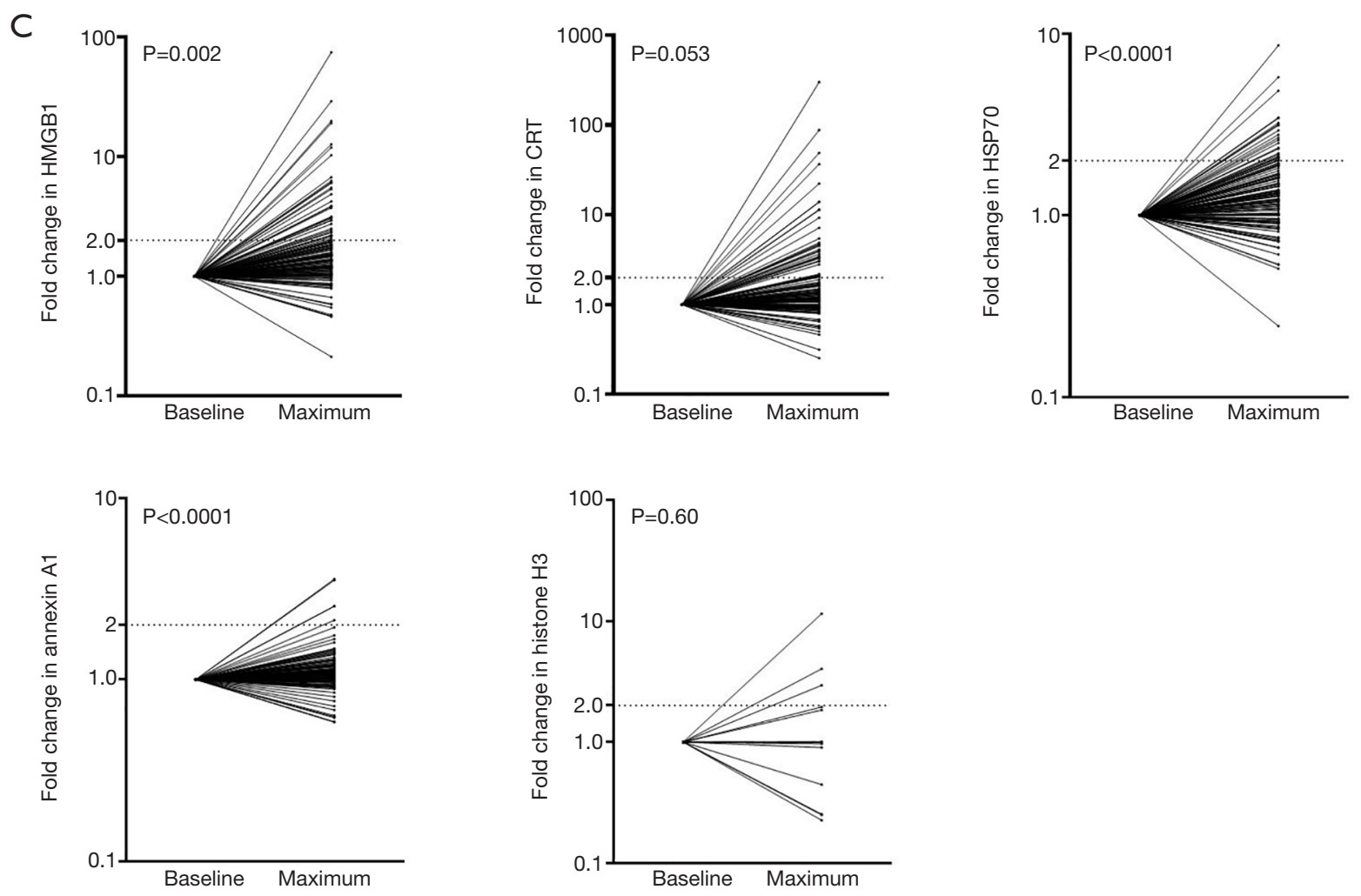

Figure 1 Dynamics of plasma DAMP levels in advanced lung cancer patients during the first cycle of systemic anticancer therapy. (A) Time course of the fold change in plasma concentrations (relative to baseline) of HMGB1, CRT, HSP70, annexin A1, and histone H3 in individual patients. Plasma samples were collected before and on days 3 and 8 after the onset of the first cycle as well as before the onset of the second cycle of treatment. (B) Fold change in the plasma levels of HMGB1, CRT, HSP70, annexin A1, and histone H3 during the first treatment cycle relative to baseline. Data are means + SEM. The P values for differences in mean values were determined by repeated-measures ANOVA. (C) Comparison of baseline values and maximum values after the onset of the first cycle of treatment for the fold change in plasma levels of the five DAMPs. The numbers of patients showing a >2-fold increase were 34, 34, 24, 5, and 3 for HMGB1, CRT, HSP70, annexin $\mathrm{A} 1$, and histone $\mathrm{H3}$, respectively. CRT and histone H3 were undetectable at baseline in 10 and 97 patients, respectively, and these patients were excluded from the analysis. The $\mathrm{P}$ values for differences in mean values were determined with the paired t test. DAMP, damageassociated molecular pattern; HMGB1, high-mobility group box 1; CRT, calreticulin; HSP70, heat shock protein 70; SEM, standard error of the mean; ANOVA, analysis of variance.

suggesting that the increased concentrations of HMGB1 and CRT in plasma may reflect the extent of ICD induction by the systemic anticancer therapy. A significant correlation was not apparent between the maximum fold changes in plasma HMGB1 and CRT levels, suggesting that the mechanisms by which HMGB1 and CRT are released from dying cancer cells may differ.

HMGB1 is a nuclear protein that associates with DNA and acts as an architectural chromatin-binding factor. During cell death, it is translocated from the nucleus to the cytoplasm and then released into the extracellular space in soluble form (23). Released HMGB1 in the extracellular environment binds to the receptor for advanced glycation end products (RAGE) and Toll-like receptors 2 and 4 and acts as a multifunctional alarmin to orchestrate immune activity (24). CRT has been implicated in various physiological and pathological processes in cells (25). The translocation of CRT from the endoplasmic reticulum to the cell surface and its consequent exposure are thought to be triggered by reactive oxygen species or endoplasmic reticulum stress (26). Extracellular CRT has also been detected at late stages of apoptosis in cancer cells as a result of passive extracellular release (27). In addition to differences in the mechanisms underlying their release 
A

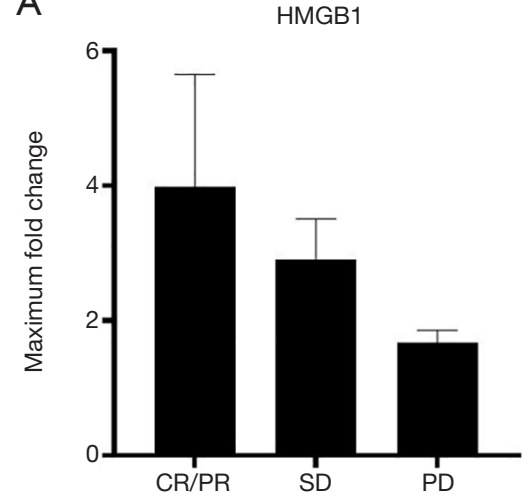

C

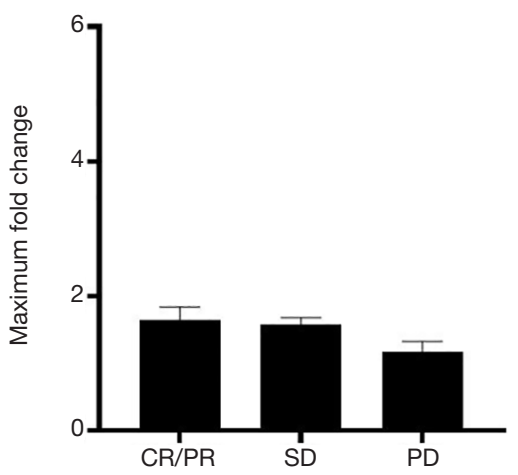

B

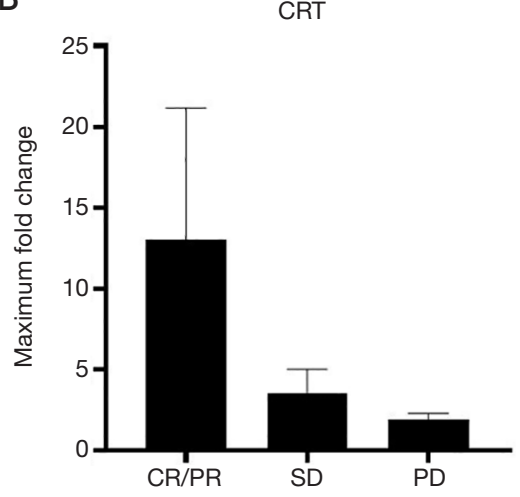

D

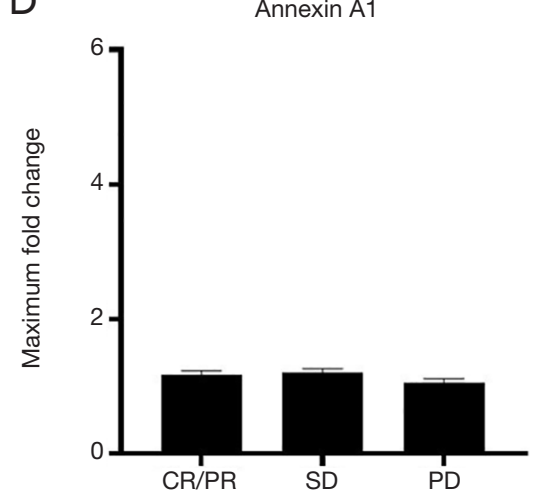

Figure 2 Association between the maximum fold change in plasma DAMP levels relative to baseline and clinical response. The maximum fold change in HMGB1 (A), CRT (B), HSP70 (C), or annexin A1 (D) levels is shown as the mean + SEM for patients classified according to tumor response. The numbers of patients who showed a CR, PR, SD or PD according to RECIST criteria were 1, 43, 63, and 13, respectively; one patient not evaluable by RECIST criteria was excluded from the analysis. CRT was undetectable at baseline in seven patients with a PR, in two with SD, and in one with $\mathrm{PD}$, and these patients were also excluded from the analysis. The $\mathrm{P}$ values for differences in mean values were determined by one-way ANOVA. DAMP, damage-associated molecular pattern; HMGB1, high-mobility group box 1; CRT, calreticulin; HSP70, heat shock protein 70; CR, complete response; PR, partial response; SD, stable disease; PD, progressive disease; RECIST, Response Evaluation Criteria in Solid Tumors; ANOVA, analysis of variance.

from dying cells, differences in the effects of the various modalities of anticancer treatment administered and in the background of the lung cancer patients studied may also have contributed to the absence of a correlation between the maximum fold changes in plasma HMGB1 and CRT levels. Although the relevance of the intervention-induced increase in the circulating concentrations of HMGB1 or CRT to systemic antitumor immunity in advanced cancer patients remains to be verified, our present findings suggest that measurement of the kinetics of HMGB1 and CRT in plasma may be a safer and more repeatable approach to the evaluation of ICD compared with the collection of tumor tissue by biopsy.

We also found that the maximum plasma levels of HSP70 and annexin A1 after the onset of systemic anticancer therapy were significantly higher than the levels at baseline. In contrast to HMGB1 and CRT, however, the maximum fold changes in plasma HSP70 and annexin A1 did not appear to be related to clinical response or the modality of anticancer treatment. It is possible that this difference may be attributable in part to the release of HSP70 and annexin A1 from normal cells such as endothelial cells (28) and macrophages (29) rather than from collapsing tumor cells. The fact that the serum levels of both HSP70 (30) and annexin A1 (31) were previously found to be significantly higher in untreated lung cancer patients than in healthy individuals may also account in part for the lack of an association between the maximum fold changes in the plasma 
A

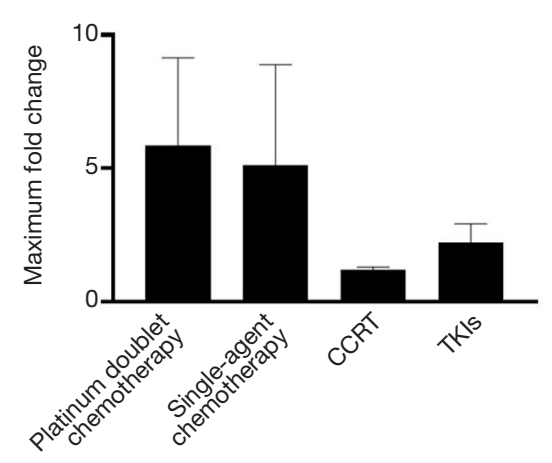

C

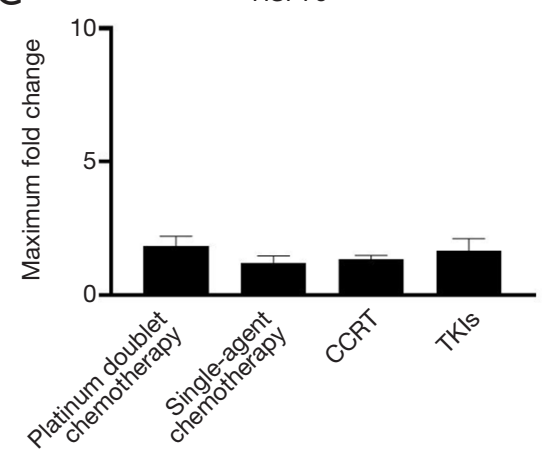

B
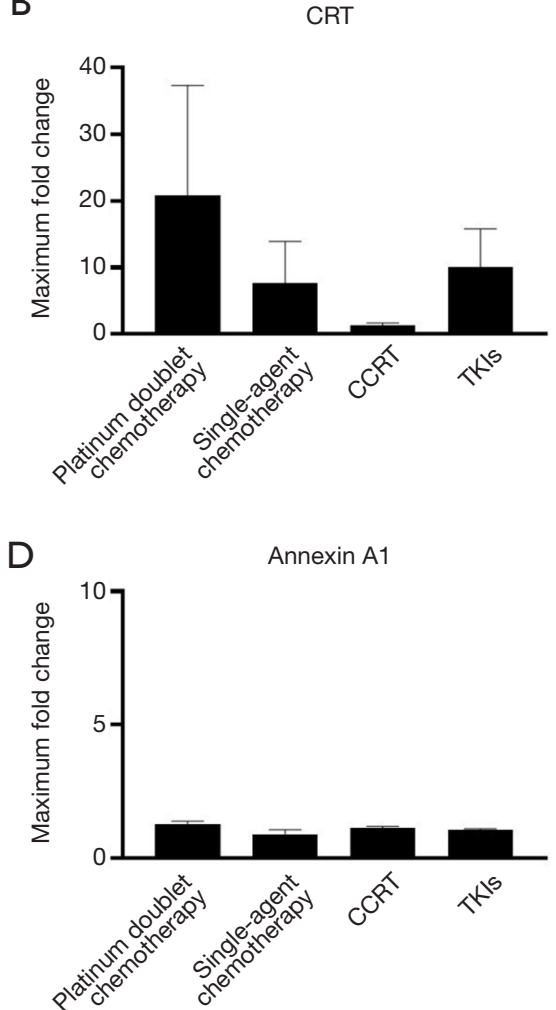

Figure 3 Maximum fold changes in plasma DAMP concentrations according to anticancer treatment modality. The maximum fold change in HMGB1 (A), CRT (B), HSP70 (C), or annexin A1 (D) levels is shown as the mean + SEM for patients with a CR ( $\mathrm{n}=1)$ or a PR ( $\mathrm{n}=43)$. Treatment modalities included platinum doublet chemotherapy ( $\mathrm{n}=22)$, single-agent chemotherapy $(\mathrm{n}=3)$, CCRT ( $\mathrm{n}=11$ ), and EGFRor ALK-TKIs $(\mathrm{n}=8)$. CRT was undetectable at baseline in four patients receiving platinum doublet chemotherapy, one receiving singleagent chemotherapy, and two receiving CCRT, and these patients were excluded from the analysis. The P values for differences in mean values were determined by one-way ANOVA. DAMP, damage-associated molecular pattern; HMGB1, high-mobility group box 1; CRT, calreticulin; HSP70, heat shock protein 70; SEM, standard error of the mean; CR, complete response; PR, partial response; CCRT, concurrent chemoradiotherapy; EGFR, epidermal growth factor receptor; ALK, anaplastic lymphoma kinase; TKI, tyrosine kinase inhibitor; ANOVA, analysis of variance.

levels of these proteins and clinical response. With regard to histone $\mathrm{H3}$, it was essentially undetectable in the plasma of most patients, suggesting that it may not be relevant to cancer biology but rather related to other morbid conditions such as infection. Indeed, one patient who achieved a partial response showed a rapid decrease in the plasma concentration of histone $\mathrm{H} 3$ between before and day 3 after the onset of the first cycle of chemotherapy. Before treatment onset, this patient had experienced infection associated with an increased C-reactive protein level and had undergone intravenous infusion of antibiotics for 7 days that led to resolution of his symptoms. The high initial circulating level of histone $\mathrm{H} 3$ in this patient may thus have been related to the infection, and its decline during systemic anticancer therapy may have reflected amelioration of the inflammatory pathophysiology of infection, consistent with a previous finding (22).

Among the therapeutic modalities administered to the study patients, we found that platinum combination chemotherapy was associated with greater maximum fold changes in the plasma levels of HMGB1 and CRT compared with CCRT or TKI therapy, suggesting that it may be superior to these latter modalities with regard to the promotion of antitumor immunity. Indeed, preclinical studies of mouse models have shown that cytotoxic chemotherapeutic agents induce ICD associated with the release of DAMPs, which then function as adjuvants 
A

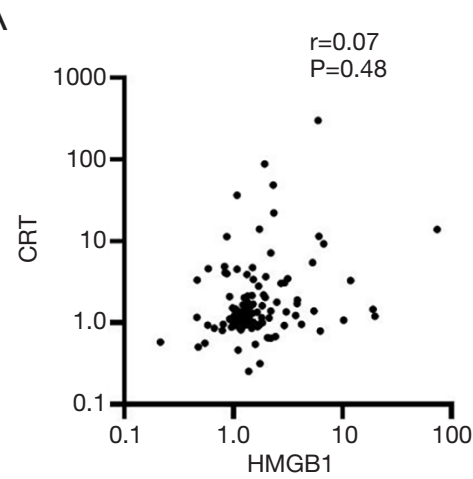

D

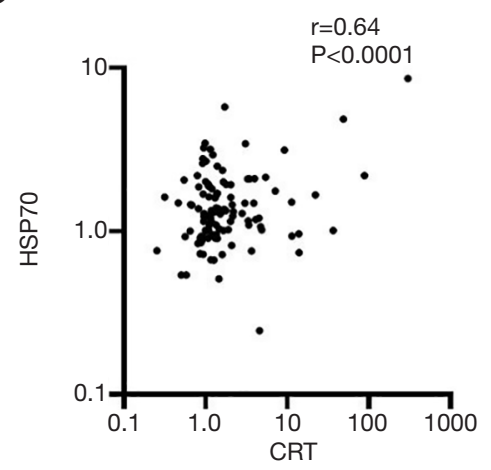

B

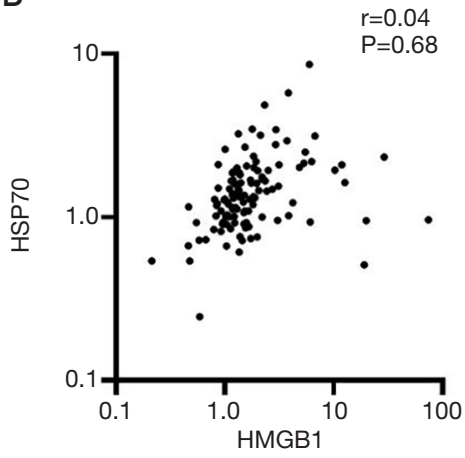

$\mathrm{E}$

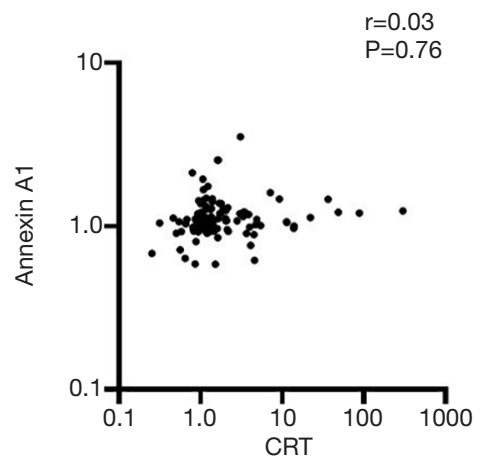

C

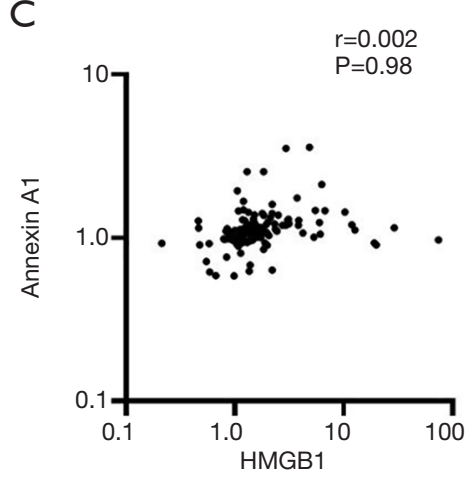

$\mathrm{F}$

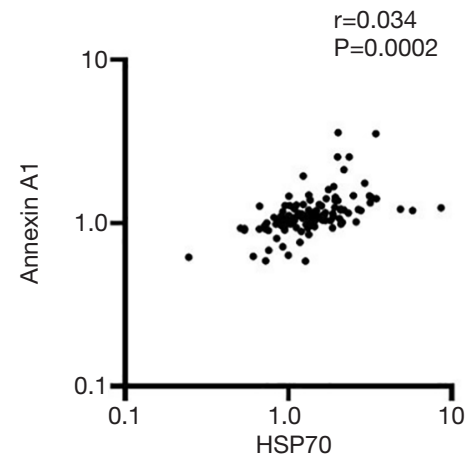

G

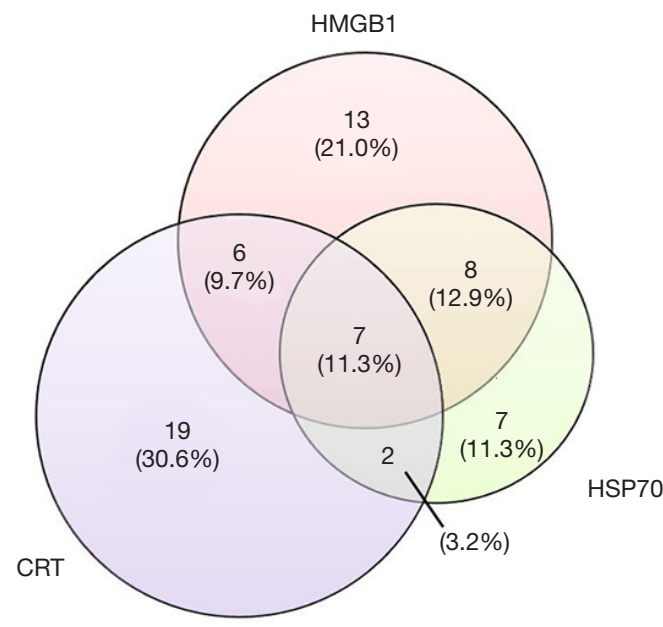

Figure 4 Correlation analysis for maximum fold changes in plasma DAMP concentrations. (A,B,C,D,E,F) Correlation analysis for HMGB1 and either CRT (A), HSP70 (B), or annexin A1 (C), for CRT and either HSP70 (D) or annexin A1 (E), or for HSP70 and annexin A1 (F) is shown. The correlation coefficient ( $\mathrm{r}$ ) and corresponding $\mathrm{P}$ values are indicated. CRT was undetectable at baseline in 10 patients, and these patients were excluded from the analysis. (G) Venn diagram for the numbers of patients showing a $>2$-fold increase in the plasma levels of HMGB1, CRT, or HSP70 during the first treatment cycle. DAMP, damage-associated molecular pattern; HMGB1, high-mobility group box 1; CRT, calreticulin; HSP70, heat shock protein 70. 
to enhance the antitumor immune response (15,32-34). Among various chemotherapeutic agents recently tested either alone or in combination, cisplatin alone was found to be most effective at inducing the release of ICD-associated DAMPs from A549 lung cancer cells in vitro, suggesting that the pairing of cisplatin with immunotherapy may be a promising treatment strategy for lung cancer (35). This previous study also suggested that derivation of a DAMP index of immunogenicity by mathematical integration might prove useful as a measure of the extent of ICD for comparison with clinical parameters such as the response to anticancer therapies (35). Our results suggest that a DAMP index based on the treatment-induced increases in the plasma levels of HMGB1, CRT, HSP70, and annexin A1 may prove helpful in this regard, although a study with a larger patient population will be necessary to evaluate this notion. Although assessment of the circulating levels of individual DAMPs is important, it will also be essential to investigate whether these levels are sufficient to activate antigen presenting cells such as dendritic cells in cancer patients during anticancer therapy.

The fact that CCRT was not associated with a substantial increase in the plasma levels of HMGB1 or CRT might be due to a relatively small extent of tumor destruction, given that CCRT is administered to patients with stage III non-small cell lung cancer, whose tumor burden is generally lower than that of those with stage IV lung cancer. Alternatively, it is possible that the time points at which plasma was collected for analysis might not have been appropriate to detect a peak in the plasma levels of HMGB1 or CRT during treatment with CCRT. Other limitations of the present study include its relatively small sample size, which may have rendered it underpowered to detect significant differences. In addition, the enrolled patients were diverse with regard to tumor histology and systemic anticancer therapy modalities.

\section{Conclusions}

In summary, we have shown that systemic anticancer therapy-in particular, platinum-based combination chemotherapy - was associated with marked increases in the plasma concentrations of HMGB1 and CRT that were apparent within the first week of treatment in patients with advanced lung cancer, and that the maximum fold changes in the circulating levels of these DAMPs tended to be associated with clinical response. Our findings suggest that patients with elevated DAMP levels in plasma who respond to anticancer therapy may experience robust ICD that boosts anticancer immunity and thereby contributes to the control of tumor growth. Serial monitoring of DAMPs in plasma during systemic anticancer therapy may be helpful for the development of novel combination regimens for cancer immunotherapy. We are currently performing a prospective observational study to investigate the relation between changes in the plasma levels of DAMPs including inflammatory cytokines and the clinical benefit of chemotherapy combined with immune checkpoint inhibitors.

\section{Acknowledgments}

We thank Akiko Sato, Shiho Isewaki, Yui Matsukuma, and Ayako Honda (Kyushu University) as well as Shingo Yamada (Shino-Test Corp.) for technical assistance.

Funding: This work was supported by the Ministry of Education, Culture, Sports, Science, and Technology of Japan (Grant-in-Aid for Scientific Research, Group C, 19K07733) and by the Japan Lung Cancer Society (Grant for Lung Cancer Research).

\section{Footnote}

Reporting Checklist: The authors have completed the STROBE reporting checklist. Available at http://dx.doi. org/10.21037/tlcr-21-92

Data Sharing Statement: Available at http://dx.doi. org/10.21037/tlcr-21-92

Peer Review File: Available at http://dx.doi.org/10.21037/ tlcr-21-92

Conflicts of Interest: All authors have completed the ICMJE uniform disclosure form (available at http:// dx.doi.org/10.21037/tlcr-21-92). IO reports grants and personal fees from Chugai Pharma, AstraZeneca, Taiho Pharmaceutical, Boehringer Ingelheim, Ono Pharmaceutical, MSD Oncology, Lilly, and Bristol-Myers Squibb; grants from Astellas Pharma, Novartis, and AbbVie; and personal fees from Pfizer outside the submitted work. The other authors have no conflicts of interest to declare.

Etbical Statement: The authors are accountable for all aspects of the work in ensuring that questions related to the accuracy or integrity of any part of the work are 
appropriately investigated and resolved. The study was conducted in accordance with the Declaration of Helsinki (as revised in 2013). It was approved by the Ethics Committee of Kyushu University and Kyushu University Hospital (IRB: 30-434), and informed consent was taken from all individual participants.

Open Access Statement: This is an Open Access article distributed in accordance with the Creative Commons Attribution-NonCommercial-NoDerivs 4.0 International License (CC BY-NC-ND 4.0), which permits the noncommercial replication and distribution of the article with the strict proviso that no changes or edits are made and the original work is properly cited (including links to both the formal publication through the relevant DOI and the license). See: https://creativecommons.org/licenses/by-nc-nd/4.0/.

\section{References}

1. Siegel RL, Miller KD, Jemal A. Cancer statistics, 2020. CA Cancer J Clin 2020;70:7-30.

2. Li XT, Yang JJ, Wu YL, et al. Toward innovative combinational immunotherapy: A systems biology perspective. Cancer Treat Rev 2018;68:1-8.

3. Gandhi L, Garassino MC. Pembrolizumab plus Chemotherapy in Lung Cancer. N Engl J Med 2018;379:e18.

4. Paz-Ares L, Luft A, Vicente D, et al. Pembrolizumab plus Chemotherapy for Squamous Non-Small-Cell Lung Cancer. N Engl J Med 2018;379:2040-51.

5. Socinski MA, Jotte RM, Cappuzzo F, et al. Atezolizumab for First-Line Treatment of Metastatic Nonsquamous NSCLC. N Engl J Med 2018;378:2288-301.

6. Horn L, Mansfield AS, Szczesna A, et al. First-Line Atezolizumab plus Chemotherapy in Extensive-Stage Small-Cell Lung Cancer. N Engl J Med 2018;379:2220-9.

7. Kroemer G, Galluzzi L, Kepp O, et al. Immunogenic cell death in cancer therapy. Annu Rev Immunol 2013;31:51-72.

8. Krysko DV, Garg AD, Kaczmarek A, et al. Immunogenic cell death and DAMPs in cancer therapy. Nat Rev Cancer 2012;12:860-75.

9. Galluzzi L, Vitale I, Warren S, et al. Consensus guidelines for the definition, detection and interpretation of immunogenic cell death. J Immunother Cancer 2020;8:e000337.

10. Inoue H, Tani K. Multimodal immunogenic cancer cell death as a consequence of anticancer cytotoxic treatments.
Cell Death Differ 2014;21:39-49.

11. Xu J, Zhang XM, Pelayo R, et al. Extracellular histones are major mediators of death in sepsis. Nat Med 2009;15:1318-21.

12. Huang H, Evankovich J, Yan W, et al. Endogenous histones function as alarmins in sterile inflammatory liver injury through Toll-like receptor 9 in mice. Hepatology 2011;54:999-1008.

13. Chen G, Emens LA. Chemoimmunotherapy: reengineering tumor immunity. Cancer Immunol Immunother 2013;62:203-16.

14. Galluzzi L, Buque A, Kepp O, et al. Immunological Effects of Conventional Chemotherapy and Targeted Anticancer Agents. Cancer Cell 2015;28:690-714.

15. Schaer DA, Geeganage S, Amaladas N, et al. The folate pathway inhibitor pemetrexed pleiotropically enhances effects of cancer immunotherapy. Clin Cancer Res 2019;25:7175-88.

16. Fucikova J, Becht E, Iribarren K, et al. Calreticulin Expression in Human Non-Small Cell Lung Cancers Correlates with Increased Accumulation of Antitumor Immune Cells and Favorable Prognosis. Cancer Res 2016;76:1746-56.

17. Suzuki Y, Mimura K, Yoshimoto Y, et al. Immunogenic tumor cell death induced by chemoradiotherapy in patients with esophageal squamous cell carcinoma. Cancer Res 2012;72:3967-76.

18. Wu W, Wang G, Tan C, et al. A sandwich enzymelinked immunosorbent assay for detection of calreticulin in human serum. Monoclon Antib Immunodiagn Immunother 2013;32:366-70.

19. Ni M, Wei W, Wang Y, et al. Serum levels of calreticulin in correlation with disease activity in patients with rheumatoid arthritis. J Clin Immunol 2013;33:947-53.

20. Liu L, Yang M, Kang R, et al. HMGB1-induced autophagy promotes chemotherapy resistance in leukemia cells. Leukemia 2011;25:23-31.

21. Ito T, Nakahara M, Masuda $Y$, et al. Circulating histone $\mathrm{H} 3$ levels are increased in septic mice in a neutrophildependent manner: preclinical evaluation of a novel sandwich ELISA for histone H3. J Intensive Care 2018;6:79.

22. Yokoyama Y, Ito T, Yasuda T, et al. Circulating histone H3 levels in septic patients are associated with coagulopathy, multiple organ failure, and death: a single-center observational study. Thromb J 2019;17:1.

23. Scaffidi P, Misteli T, Bianchi ME. Release of chromatin protein HMGB1 by necrotic cells triggers inflammation. 
Nature 2002;418:191-5.

24. Diener KR, Al-Dasooqi N, Lousberg EL, et al. The multifunctional alarmin HMGB1 with roles in the pathophysiology of sepsis and cancer. Immunol Cell Biol 2013;91:443-50

25. Lu YC, Weng WC, Lee H. Functional roles of calreticulin in cancer biology. Biomed Res Int 2015;2015:526524.

26. Panaretakis T, Kepp O, Brockmeier U, et al. Mechanisms of pre-apoptotic calreticulin exposure in immunogenic cell death. EMBO J 2009;28:578-90.

27. Garg AD, Krysko DV, Vandenabeele P, et al. Hypericinbased photodynamic therapy induces surface exposure of damage-associated molecular patterns like HSP70 and calreticulin. Cancer Immunol Immunother 2012;61:215-21.

28. McArthur S, Loiola RA, Maggioli E, et al. The restorative role of annexin A1 at the blood-brain barrier. Fluids Barriers CNS 2016;13:17.

29. Maderna P, Yona S, Perretti M, et al. Modulation of phagocytosis of apoptotic neutrophils by supernatant from dexamethasone-treated macrophages and annexin-derived peptide Ac(2-26). J Immunol 2005;174:3727-33.

30. Ren B, Luo S, Xu F, et al. The expression of DAMP

Cite this article as: Inoue $\mathrm{H}$, Tsutsumi $\mathrm{H}$, Tanaka $\mathrm{K}$, Iwama E, Shiraishi Y, Hirayama A, Nakanishi T, Ando H, Nakajima M, Shinozaki S, Ogata H, Uryu K, Okamura K, Kimura S, Ogawa T, Ota K, Yoneshima Y, Hamada N, Nakanishi Y, Okamoto I. Increased plasma levels of damage-associated molecular patterns during systemic anticancer therapy in patients with advanced lung cancer. Transl Lung Cancer Res 2021;10(6):2475-2486. doi: 10.21037/ tlcr-21-92 proteins HSP70 and cancer-testis antigen SPAG9 in peripheral blood of patients with HCC and lung cancer. Cell Stress Chaperones 2017;22:237-44.

31. Rong B, Zhao C, Liu H, et al. Elevated serum annexin A1 as potential diagnostic marker for lung cancer: a retrospective case-control study. Am J Transl Res 2014;6:558-69.

32. Pfirschke C, Engblom C, Rickelt S, et al. Immunogenic Chemotherapy Sensitizes Tumors to Checkpoint Blockade Therapy. Immunity 2016;44:343-54.

33. Apetoh L, Ghiringhelli F, Tesniere A, et al. Toll-like receptor 4-dependent contribution of the immune system to anticancer chemotherapy and radiotherapy. Nat Med 2007;13:1050-9.

34. Vandenabeele P, Vandecasteele K, Bachert C, et al. Immunogenic Apoptotic Cell Death and Anticancer Immunity. Adv Exp Med Biol 2016;930:133-49.

35. Solari JIG, Filippi-Chiela E, Pilar ES, et al. Damageassociated molecular patterns (DAMPs) related to immunogenic cell death are differentially triggered by clinically relevant chemotherapeutics in lung adenocarcinoma cells. BMC Cancer 2020;20:474. 

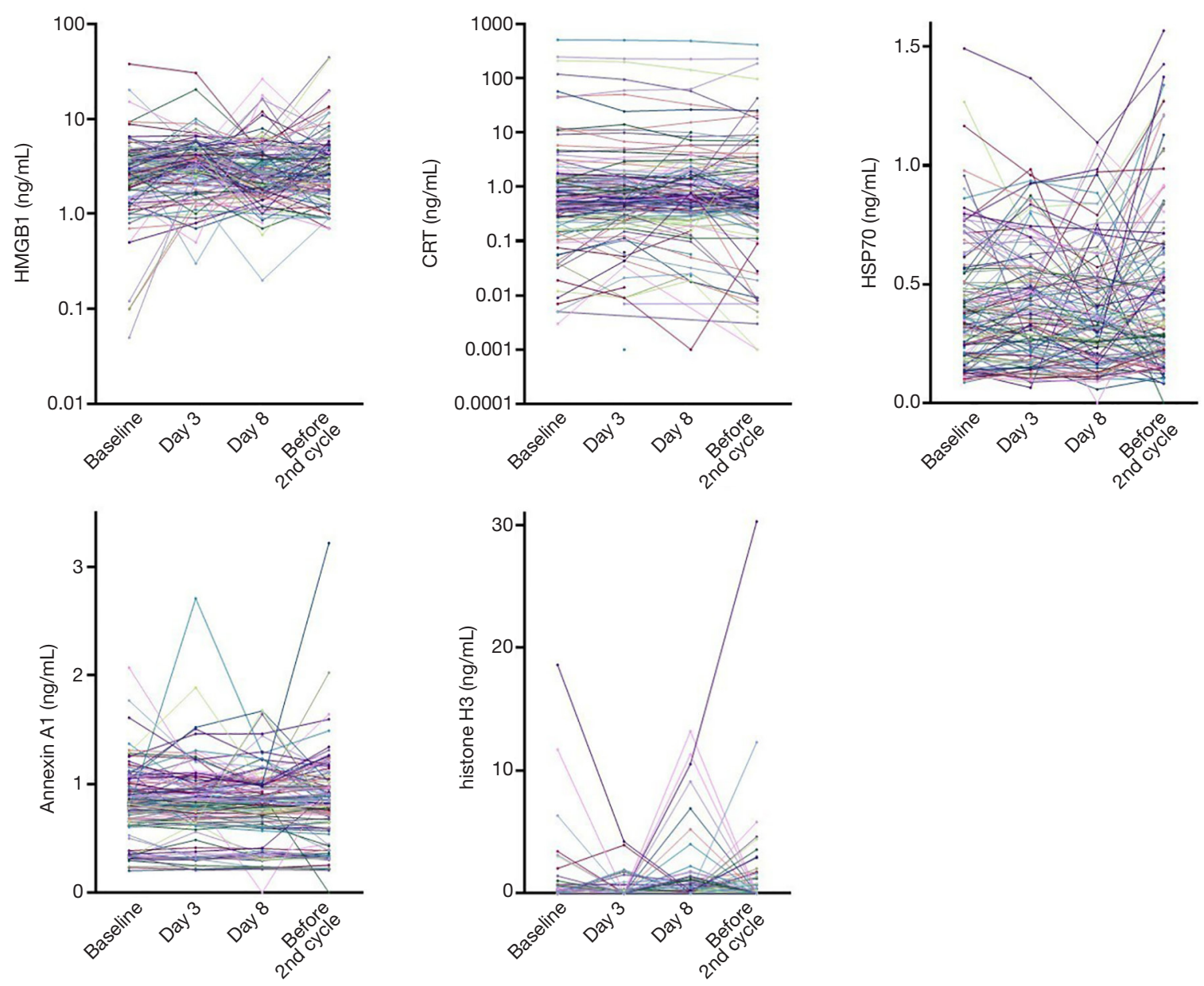

Figure S1 Dynamics of plasma DAMP levels in advanced lung cancer patients during the first cycle of systemic anticancer therapy. Time courses of the plasma concentrations of HMGB1, CRT, HSP70, annexin A1, and histone H3 in individual patients are shown. Plasma samples were collected before and on days 3 and 8 after the onset of the first cycle as well as before the onset of the second cycle of treatment. DAMP, damage-associated molecular pattern; HMGB1, high-mobility group box 1; CRT, calreticulin; HSP70, heat shock protein 70 . 